\title{
Minimizing Time-to-Rank: A Learning and Recommendation Approach
}

\author{
Haoming Li ${ }^{1}$, Sujoy Sikdar ${ }^{2}$, Rohit Vaish ${ }^{2 *}$, Junming Wang ${ }^{2}$, Lirong Xia ${ }^{2}$ and Chaonan Ye \\ ${ }^{1}$ Duke University \\ ${ }^{2}$ Rensselaer Polytechnic Institute \\ ${ }^{3}$ Stanford University
}

haoming.li@duke.edu, \{sikdas, vaishr2, wangj33\}@rpi.edu, xial@cs.rpi.edu, canonyeee@gmail.com

\begin{abstract}
Consider the following problem faced by an online voting platform: A user is provided with a list of alternatives, and is asked to rank them in order of preference using only drag-and-drop operations. The platform's goal is to recommend an initial ranking that minimizes the time spent by the user in arriving at her desired ranking. We develop the first optimization framework to address this problem, and make theoretical as well as practical contributions. On the practical side, our experiments on Amazon Mechanical Turk provide two interesting insights about user behavior: First, that users' ranking strategies closely resemble selection or insertion sort, and second, that the time taken for a drag-and-drop operation depends linearly on the number of positions moved. These insights directly motivate our theoretical model of the optimization problem. We show that computing an optimal recommendation is NP-hard, and provide exact and approximation algorithms for a variety of special cases of the problem. Experimental evaluation on MTurk shows that, compared to a random recommendation strategy, the proposed approach reduces the average time-to-rank by up to $50 \%$.
\end{abstract}

\section{Introduction}

Eliciting preferences in the form of rankings over a set of alternatives is a common task in social choice, crowdsourcing, and in daily life. For example, the organizer of a meeting might ask the participants to rank a set of time-slots based on their individual schedules. Likewise, in an election, voters might be required to rank a set of candidates in order of preference.

Over the years, computerized systems have been increasingly used in carrying out preference elicitation tasks such as the ones mentioned above. Indeed, recently there has been a proliferation of online voting platforms such as CIVS, OPRA, Pnyx, RoboVote, and Whale ${ }^{4}{ }^{1}$ In many of these platforms,

\footnotetext{
${ }^{*}$ Contact Author

${ }^{1}$ CIVS (https://civs.cs.cornell.edu/), OPRA (opra.io), Pnyx (https://pnyx.dss.in.tum.de/), RoboVote (http://robovote.org/), Whale $^{4}$ (https://whale.imag.fr/).
}

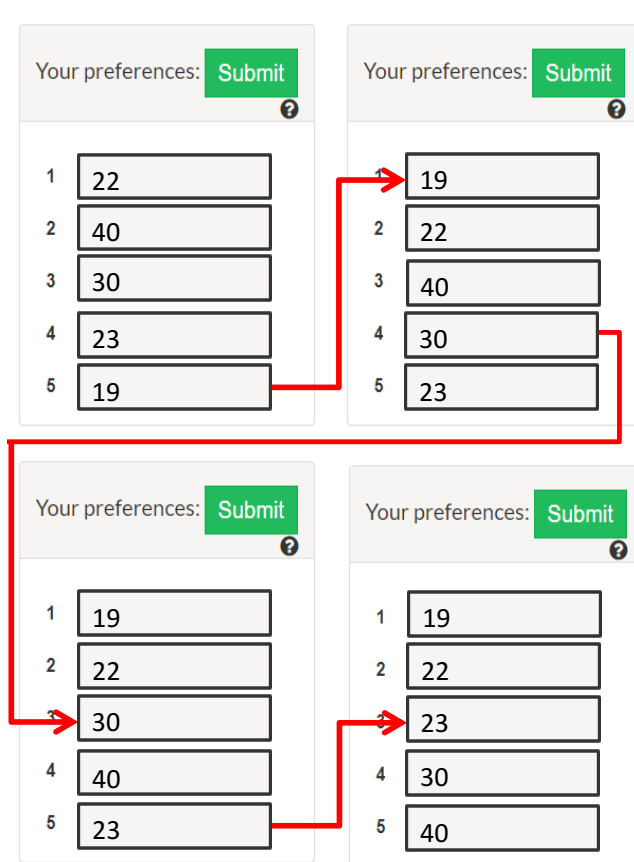

Figure 1: Sorting via drag-and-drop operations.

a user is presented with an arbitrarily ordered list of alternatives, and is asked to shuffle them around in-place using drag-and-drop operations until her desired preference ordering is achieved. Figure 1 illustrates the use of drag-and-drop operations to sort a list of numbers in descending order.

Our focus in this work is on time-to-rank, i.e., the time it takes for a user to arrive at her desired ranking, starting from a ranking suggested by the platform and using only drag-anddrop operations. We study this problem from the perspective of the voting platform that wants to recommend an optimal initial ranking to the user (i.e., one that minimizes time-to-rank). Time to accomplish a designated task is widely considered as a key consideration in the usability of automated systems [Bevan et al., 2015; Albert and Tullis, 2013], and serves as a proxy for user effort. Indeed, 'time on task' was identified as a key factor in the usability and efficiency of computerized voting systems in a 2004 report by NIST to the U.S. Congress for the Help America Vote Act (HAVA) [Laskowski et al., 2004]. 


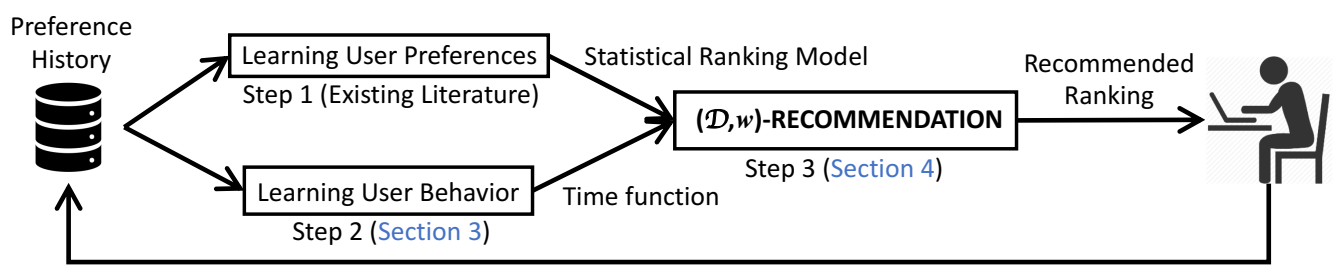

Figure 2: High level overview of our framework. Our technical contributions are highlighted in blue.

Note that the initial ranking suggested by the platform can have a significant impact on the time spent by the user on the ranking task. Indeed, if the user's preferences are known beforehand, then the platform can simply recommended it to her and she will only need to verify that the ordering is correct. In practice, however, users' preferences are often unknown. Furthermore, users employ a wide variety of ranking strategies, and based on their proficiency with the interface, users can have very different drag-and-drop times. All these factors make the task of predicting the time-to-rank and finding an optimal recommendation challenging and non-trivial.

We emphasize the subtle difference between our problem and that of preference elicitation. The latter involves repeatedly asking questions to the users (e.g., pairwise comparisons between alternatives) to gather enough information about their preferences. By contrast, our problem involves a one-shot recommendation followed by a series of drag-and-drop operations until the desired ranking is achieved. There is an extensive literature on preference elicitation [Conitzer and Sandholm, 2002; Blum et al., 2004; Boutilier, 2013; Busa-Fekete et al., 2014; Soufiani et al., 2013; Zhao et al., 2018]. Yet, somewhat surprisingly, the problem of recommending a ranking that minimizes users' time has received little attention. Our work aims to address this gap.

\section{Our Contributions}

We make contributions on three fronts:

- On the conceptual side, we propose the problem of minimizing time-to-rank and outline a framework for addressing it (Figure 2).

- On the theoretical side, we formulate the optimization problem of finding a recommendation to minimize timeto-rank (Section 4). We show that this problem is NPhard, even under highly restricted settings (Theorem 3). We complement the intractability results by providing a number of exact (Theorem 2) and approximation algorithms (Theorems 4 to 6) for special cases of the problem.

- We use experimental analysis to motivate our modeling assumptions as well as to justify the effectiveness of our approach (Section 5). Our experiments on MTurk reveal two insights about user behavior (Section 5.1): (1) The ranking strategies of real-world users closely resemble insertion/selection sort, and (2) the drag-and-drop time of an alternative varies linearly with the distance moved. We find that a simple adaptive strategy (based on Borda count voting rule) can reduce time-to-rank by up to $50 \%$ compared to a random strategy (Section 5.2), validating the usefulness of the proposed framework.

\subsection{Overview of Our Framework}

Figure 2 illustrates the proposed framework which consists of three key steps. In Step 1, we learn user preferences from historical data by developing a statistical ranking model, typically in the form of a distribution $\mathcal{D}$ over the space of all rankings (refer to Section 2 for examples of ranking models). In Step 2 , which runs in parallel to Step 1, we learn user behavior; in particular, we identify their sorting strategies (Section 3.1) as well as their drag-and-drop times (Section 3.2). Together, these two components define the $t$ ime function which models the time taken by a user in transforming a given initial ranking $\sigma$ into a target ranking $\tau$, denoted by time $(\sigma, \tau)$. The ranking model $\mathcal{D}$ from Step 1 and the time function from Step 2 together define the recommendation problem in Step 3, called $(\mathcal{D}, \mathbf{w})$-RECOMMENDATION (the parameter $\mathbf{w}$ is closely related to the time function; we elaborate on this below). This is the optimization problem of computing a ranking $\sigma$ that minimizes the expected time-to-rank of the user, i.e., minimizes $\mathbb{E}_{\tau \sim \mathcal{D}}[\operatorname{time}(\sigma, \tau)]$. The user is then recommended $\sigma$, and her preference history is updated.

The literature on learning statistical ranking models is already well-developed [Guiver and Snelson, 2009; Awasthi et al., 2014; Lu and Boutilier, 2014; Zhao et al., 2016; Xia, 2019]. Thus, while this is a key ingredient of our framework (Step 1), our work only focuses on Steps 2 and 3 concerning user behavior and the recommendation problem.

Recall that the $t$ ime function defines the time taken by a user in transforming a given ranking $\sigma$ into a target ranking $\tau$. For a user who follows a fixed sorting algorithm (e.g., insertion or selection sort), the $t$ ime function can be broken down into (1) the number of drag-and-drop operations suggested by the sorting algorithm, and, (2) the (average) time taken for each drag-and-drop operation by the user. As we will show in Lemma 1 in Section 3.1, point (1) above is independent of the choice of the sorting algorithm. Therefore, the time function can be equivalently defined in terms of the weight function $\mathbf{w}$, which describes the time taken by a user, denoted by $\mathrm{w}(\ell)$, in moving an alternative by $\ell$ positions via a drag-and-drop operation. For this reason, we use $\mathrm{w}$ in the formulation of $(\mathcal{D}, \mathbf{w})$-RECOMMENDATION.

Applicability. Our framework is best suited for users who have already formed their preferences, so that the recommended ranking does not bias their preferences. This is a natural assumption in some applications, such as in the meeting organization example in Section 1. In general, however, it is possible that a user, who is undecided between options $A$ and $B$, might prefer $A$ over $B$ if presented in that order by the 
Proceedings of the Twenty-Eighth International Joint Conference on Artificial Intelligence (IJCAI-19)

\begin{tabular}{cccccc}
\hline \multirow{2}{*}{ Distribution $\mathcal{D}$} & \multicolumn{3}{c}{ Linear Weights } & & General Weights \\
\cline { 2 - 4 } \cline { 3 - 5 }$k$-mixture Plackett-Luce $(k$-PL) & Hardness & Exact Algo. & Approx. Algo. & Approx. Algo. \\
\hline \multirow{2}{*}{$k$-mixture Mallows $(k$-MM) } & $\begin{array}{c}\text { NP-c even for } k=4 \\
\text { (Theorem 3) }\end{array}$ & $\begin{array}{c}\text { Poly for } k=1 \\
\text { (Theorem 2) } k=4\end{array}$ & $\begin{array}{c}\text { PTAS (Theorem 4) } \\
\text { 5-approx. (Theorem 5) }\end{array}$ & $\begin{array}{c}\alpha \beta \text {-approx. } \\
(\text { Theorem 6) }\end{array}$ \\
\hline Uniform (Unif) & $\begin{array}{c}\text { NP-c even for } n=4=1 \\
\text { (Theorem 3) }\end{array}$ & $\begin{array}{c}\text { Poly for } n \in\{1,2\} \\
\text { (Theorem 2) }\end{array}$ & $\begin{array}{c}\text { PTAS (Theorem 4) } \\
\text { 5-approx. (Theorem 5) }\end{array}$ & $\begin{array}{c}\alpha \beta \text {-approx. } \\
\text { (Theorem 6) }\end{array}$ \\
\hline
\end{tabular}

Table 1: Computational complexity results for $(\mathcal{D}, \mathbf{w})$-RECOMMENDATION. Each row corresponds to a preference model and each column corresponds to a weight function. We use the shorthands Poly, NP-c, PTAS, and $\alpha \beta$-approx. to denote polynomial-time (exact) algorithm, NPcomplete, polynomial-time approximation scheme, and $\alpha \beta$-approximation algorithm (for $(\alpha, \beta)$-close weights; see Definition 6) respectively.

recommended ranking. Investigating such biases (a.k.a. "framing effect") is an interesting direction for future work.

Additional related work. Our work is related to the literature on inferring a ground truth ordering from noisy information [Braverman and Mossel, 2008], and aggregating preferences by minimizing some notion of distance to the observed rankings such as the total Kendall's Tau distance [Procaccia and Shah, 2016]. Previous work on preference learning and learning to rank can also be integrated in our framework [Liu, 2011; Lu and Boutilier, 2014; Khetan and Oh, 2016; Agarwal, 2016; Negahban et al., 2017; Zhao and Xia, 2018].

\section{Preliminaries}

Let $A=\left\{a_{1}, \ldots, a_{m}\right\}$ denote a set of $m$ alternatives, and let $\mathcal{L}(A)$ be the set of all linear orders over $A$. For any $\sigma \in \mathcal{L}(A)$, $a_{i} \succ_{\sigma} a_{j}$ denotes that $a_{i}$ is preferred over $a_{j}$ under $\sigma$, and let $\sigma(k)$ denote the $k^{\text {th }}$ most preferred alternative in $\sigma$. A set of $n$ linear orders $\left\{\sigma^{(1)}, \ldots, \sigma^{(n)}\right\}$ is called a preference profile.

Definition 1 (Kendall's Tau distance; [Kendall, 1938]). Given two linear orders $\sigma, \sigma^{\prime} \in \mathcal{L}(A)$, the Kendall's Tau distance $\mathrm{d}_{\mathrm{kt}}\left(\sigma, \sigma^{\prime}\right)$ is the number of pairwise disagreements between $\sigma$ and $\sigma^{\prime}$. That is, $\mathrm{d}_{\mathrm{kt}}\left(\sigma, \sigma^{\prime}\right):=\sum_{a_{i}, a_{j} \in A} \mathbf{1}\left[a_{j} \succ_{\sigma^{\prime}}\right.$ $a_{i}$ and $\left.a_{i} \succ_{\sigma} a_{j}\right]$, where 1 is the indicator function.

Definition 2 (Plackett-Luce model; [Plackett, 1975; Luce, 1959]). Let $\theta:=\left(\theta_{1}, \ldots, \theta_{m}\right)$ be such that $\theta_{i} \in(0,1)$ for each $i \in[m]$ and $\sum_{i \in[m]} \theta_{i}=1$. Let $\Theta$ denote the corresponding parameter space. The Plackett-Luce (PL) model parameterized by $\theta \in \Theta$ defines a distribution over the set of linear orders $\mathcal{L}(A)$ as follows: The probability of generating $\sigma:=\left(a_{i_{1}} \succ a_{i_{2}} \succ \ldots \succ a_{i_{m}}\right)$ is given by

$$
\operatorname{Pr}(\sigma \mid \theta)=\frac{\theta_{i_{1}}}{\sum_{\ell=1}^{m} \theta_{i_{\ell}}} \cdot \frac{\theta_{i_{2}}}{\sum_{\ell=2}^{m} \theta_{i_{\ell}}} \cdots \frac{\theta_{i_{m-1}}}{\theta_{i_{m-1}}+\theta_{i_{m}}} .
$$

More generally, a k-mixture Plackett-Luce model ( $k$-PL) is parameterized by $\left\{\gamma^{(\ell)}, \theta^{(\ell)}\right\}_{\ell=1}^{k}$, where $\sum_{\ell=1}^{k} \gamma^{(\ell)}=1$, $\gamma^{(\ell)} \geq 0$ for all $\ell \in[k]$, and $\theta^{(\ell)} \in \Theta$ for all $\ell \in$ $[k]$. The probability of generating $\sigma \in \mathcal{L}(A)$ is given by $\operatorname{Pr}\left(\sigma \mid\left\{\gamma^{(\ell)}, \theta^{(\ell)}\right\}_{\ell=1}^{k}\right)=\sum_{\ell=1}^{k} \gamma^{(\ell)} \operatorname{Pr}\left(\sigma \mid \theta^{(\ell)}\right)$.

Definition 3 (Mallows model; [Mallows, 1957]). The Mallows model (MM) is specified by a reference ranking $\sigma^{*} \in$
$\mathcal{L}(A)$ and a dispersion parameter $\phi \in(0,1]$. The probability of generating a ranking $\sigma$ is given by $\operatorname{Pr}\left(\sigma \mid \sigma^{*}, \phi\right)=\frac{\phi^{\mathrm{d}_{\mathrm{kt}}\left(\sigma, \sigma^{*}\right)}}{Z}$, where $Z=\sum_{\sigma^{\prime} \in \mathcal{L}(A)} \phi^{\mathrm{d}_{\mathrm{kt}}\left(\sigma^{\prime}, \sigma^{*}\right)}$.

More generally, a k-mixture Mallows model ( $k$-MM) is parameterized by $\left\{\gamma^{(\ell)}, \sigma_{(\ell)}^{*}, \phi_{(\ell)}\right\}_{\ell=1}^{k}$, where $\sum_{\ell=1}^{k} \gamma^{(\ell)}=1$, $\gamma^{(\ell)} \geq 0$ for all $\ell \in[k]$, and $\sigma_{(\ell)}^{*} \in \mathcal{L}(A), \phi_{(\ell)} \in(0,1]$ for all $\ell \in[k]$. The probability of generating $\sigma \in \mathcal{L}(A)$ is given by $\operatorname{Pr}\left(\sigma \mid\left\{\gamma^{(\ell)}, \sigma_{(\ell)}^{*}, \phi_{(\ell)}\right\}_{\ell=1}^{k}\right)=\sum_{\ell=1}^{k} \gamma^{(\ell)} \operatorname{Pr}\left(\sigma \mid \sigma_{(\ell)}^{*}, \phi_{(\ell)}\right)$.

Definition 4 (Uniform distribution). Under the uniform distribution (Unif) supported on a preference profile $\left\{\sigma^{(i)}\right\}_{i=1}^{n}$, the probability of generating $\sigma \in \mathcal{L}(A)$ is $\frac{1}{n}$ if $\sigma \in\left\{\sigma^{(i)}\right\}_{i=1}^{n}$ and 0 otherwise.

\section{Modeling User Behavior}

In this section, we will model the time spent by the user in transforming the recommended ranking $\sigma$ into the target ranking $\tau$. Our formulation involves the sorting strategy of the user (Section 3.1) as well as her drag-and-drop time (Section 3.2).

\subsection{Sorting Algorithms}

A sorting algorithm takes as input a ranking $\sigma \in \mathcal{L}(A)$ and performs a sequence of drag-and-drop operations until the target ranking is achieved. At each step, an alternative is moved from its current position to another (possibly different) position and the current ranking is updated accordingly. Below we will describe two well-known examples of sorting algorithms: selection sort and insertion sort. Let $\sigma^{(k)}$ denote the current list at time step $k \in\{1,2, \ldots\}$ (i.e., before the sorting operation at time step $k$ takes place). Thus, $\sigma^{(1)}=\sigma$. For any $\sigma \in \mathcal{L}(A)$, define the $k$-prefix set of $\sigma$ as $P_{k}(\sigma):=\{\sigma(1), \sigma(2), \ldots, \sigma(k)\}$ (where $P_{0}(\sigma):=\emptyset$ ) and corresponding suffix set as $S_{k}(\sigma):=A \backslash P_{k}(\sigma)$.

Selection sort. Let $a_{i}$ denote the most preferred alternative according to $\tau$ in the set $S_{k-1}\left(\sigma^{(k)}\right)$. At step $k$ of selection sort, the alternative $a_{i}$ is promoted to a position such that the top $k$ alternatives in the new list are ordered according to $\tau$. Note that this step is well-defined only under the sorted-prefix property, i.e., at the beginning of step $k$ of the algorithm, the alternatives in $P_{k-1}\left(\sigma^{(k)}\right)$ are sorted according to $\tau$. This property is maintained by selection sort. 
Insertion sort. Let $a_{i}$ denote the most preferred alternative in $S_{k-1}\left(\sigma^{(k)}\right)$ according to $\sigma^{(k)}$. At step $k$ of insertion sort, the alternative $a_{i}$ is promoted to a position such that the top $k$ alternatives in the new list are ordered according to $\tau$. Note that this step is well-defined only under the sorted-prefix property, which is maintained by insertion sort.

Sorting algorithms. In this work, we will be concerned with sorting algorithms that involve a combination of insertion and selection sort. Specifically, we will use the term sorting algorithm to refer to a sequence of steps $s_{1}, s_{2}, \ldots$ such that each step $s_{k}$ corresponds to either selection or insertion sort, i.e., $s_{k} \in\{$ SEL,INS $\}$ for every $k$. If $s_{k}=$ SEL, then the algorithm promotes the most preferred alternative in $S_{k-1}\left(\sigma^{(k)}\right)$ (according to $\tau$ ) to a position such that the top $k$ alternatives in the new list are ordered according to $\tau$. If $s_{k}=$ INS, then the algorithm promotes the most preferred alternative in $S_{k-1}\left(\sigma^{(k)}\right)$ (according to $\sigma^{(k)}$ ) to a position such that the top $k$ alternatives in the new list are ordered according to $\tau$. For example, in Figure 1, starting from the recommended list at the extreme left, the user performs a selection sort operation (promoting 19 to the top of the current list) followed by an insertion sort operation (promoting 30 to its correct position in the sorted prefix $\{19,22,40\}$ ) followed by either selection or insertion sort operation (promoting 23 to its correct position). We will denote a generic sorting algorithm by $\mathcal{A}$ and the class of all sorting algorithms by $\mathfrak{A}$.

Count function. Given a sorting algorithm $\mathcal{A}$, a source ranking $\sigma \in \mathcal{L}(A)$ and a target ranking $\tau \in \mathcal{L}(A)$, the count function $f_{\mathcal{A}}^{\sigma \rightarrow \tau}:[m-1] \rightarrow \mathbb{Z}_{+} \cup\{0\}$ keeps track of the number of drag-and-drop operations (and the number of positions by which some alternative is moved in each such operation) during the execution of $\mathcal{A}$. Formally, $f_{\mathcal{A}}^{\sigma \rightarrow \tau}(\ell)$ is the number of times some alternative is 'moved up by $\ell$ positions' during the execution of algorithm $\mathcal{A}$ when the source and target rankings are $\sigma$ and $\tau$ respectively. ${ }^{2}$ For example, let $\mathcal{A}$ be insertion sort, $\sigma=(d, c, a, b)$, and $\tau=(a, b, c, d)$. In step 1 , the user considers the alternative $d$ and no move-up operation is required. In step 2, the user promotes $c$ by one position (since $\left.c \succ_{\tau} d\right)$ to obtain the new list $(c, d, a, b)$. In step 3, the user promotes $a$ by two positions to obtain $(a, c, d, b)$. Finally, the user promotes $b$ by two positions to obtain the target list $(a, b, c, d)$. Overall, the user performs one 'move up by one position' operation and two 'move up by two positions' operations. Hence, $f_{\mathcal{A}}^{\sigma \rightarrow \tau}(1)=1, f_{\mathcal{A}}^{\sigma \rightarrow \tau}(2)=2$, and $f_{\mathcal{A}}^{\sigma \rightarrow \tau}(3)=0$. We will write \#moves to denote the total number of drag-and-drop operations performed during the execution of $\mathcal{A}$, i.e., \#moves $=\sum_{\ell=1}^{m-1} f_{\mathcal{A}}^{\sigma \rightarrow \tau}(\ell)$.

Remark 1. Notice the difference between the number of dragand-drop operations (\#moves) and the total distance covered (i.e., the number of positions by which alternatives are moved). Indeed, the above example involves three drag-anddrop operations (\#moves $=3$ ), but the total distance moved is

\footnotetext{
${ }^{2}$ Notice that we do not keep track of which alternative is moved by $\ell$ positions. We believe it is reasonable to assume that moving $a_{1}$ up by $\ell$ positions takes the same time as it does for $a_{2}$. Also, we do not need to define the count function for move down operations as neither selection sort nor insertion sort will ever make such a move.
}

$0+1+2+2=5$. The latter quantity is equal to $\mathrm{d}_{\mathrm{kt}}(\sigma, \tau)$.

Lemma 1. For any two sorting algorithms $\mathcal{A}, \mathcal{A}^{\prime} \in \mathfrak{A}$, any $\sigma, \tau \in \mathcal{L}(A)$, and any $\ell \in[m-1], f_{\mathcal{A}}^{\sigma \rightarrow \tau}(\ell)=f_{\mathcal{A}^{\prime}}^{\sigma \rightarrow \tau}(\ell)$.

In light of Lemma 1 , we will hereafter drop the subscript $\mathcal{A}$ and simply write $f^{\sigma \rightarrow \tau}$ instead of $f_{\mathcal{A}}^{\sigma \rightarrow \tau}$. All missing proofs can be found in the full version [Li et al., 2019].

\subsection{Drag-and-Drop Time}

Weight function. The weight function w : $[m-1] \rightarrow \mathbb{R}_{\geq 0}$ models the time taken for each drag-and-drop operation; specifically, w $(\ell)$ denotes the time taken by the user in moving an alternative up by $\ell$ positions. ${ }^{3}$ Of particular interest to us will be the linear weight function $\mathrm{w}_{\operatorname{lin}}(\ell)=\ell$ for each $\ell \in[m-1]$ and the affine weight function $\mathbf{w}_{\text {aff }}(\ell)=c \ell+d$ for each $\ell \in[m-1]$ and fixed constants $c, d \in \mathbb{N}$.

Time function. Given the count function $f^{\sigma \rightarrow \tau}$ and the weight function $\mathrm{w}$, the time function is defined as their inner product, i.e., time $\mathbf{w}_{\mathbf{w}}(\sigma, \tau)=\left\langle f^{\sigma \rightarrow \tau}, \mathbf{w}\right\rangle=$ $\sum_{\ell=1}^{m-1} f^{\sigma \rightarrow \tau}(\ell) \cdot \mathbf{w}(\ell)$.

Theorem 1 shows that for the linear weight function $\mathbf{w}_{\text {lin }}$, time is equal to the Kendall's Tau distance, and for the affine weight function, time is equal to a weighted combination of Kendall's Tau distance and the total number of moves.

Theorem 1. For any $\sigma, \tau \in \mathcal{L}(A)$, time w $_{\text {lin }}(\sigma, \tau)=$ $\mathrm{d}_{\mathrm{kt}}(\sigma, \tau)$ and time $\mathbf{w}_{\text {aff }}(\sigma, \tau)=c \cdot \mathrm{d}_{\mathrm{kt}}(\sigma, \tau)+d \cdot$ \#moves.

\section{Formulation of the Recommendation Problem and Theoretical Results}

We model the recommendation problem as the following computational problem: Given the preference distribution $\mathcal{D}$ of the user and her time function (which, in turn, is determined by the weight function $w$ ), find a ranking that minimizes the expected time taken by the user to transform the recommended ranking $\sigma$ into her preference $\tau$.

Definition 5 (( $\mathcal{D}, \mathbf{w})$-RECOMMENDATION). Given a distribution $\mathcal{D}$ over $\mathcal{L}(A)$, a weight function $\mathbf{w}$, and a number $\delta \in \mathbb{Q}$, does there exist $\sigma \in \mathcal{L}(A)$ so that $\mathbb{E}_{\tau \sim \mathcal{D}}\left[\operatorname{time} \mathrm{w}_{\mathbf{w}}(\sigma, \tau)\right] \leq \delta$ ?

We will focus on settings where $\mathcal{D}$ is Plackett-Luce, Mallows, or Uniform, and $\mathbf{w} \in\{$ Linear, Affine, General $\}$. Note that if the quantity $\mathbb{E}_{\tau \sim \mathcal{D}}\left[\operatorname{time}_{\mathbf{w}}(\sigma, \tau)\right]$ can be computed in polynomial time for a given distribution $\mathcal{D}$ and weight function $\mathbf{w}$, then $(\mathcal{D}, \mathbf{w})$-RECOMMENDATION is in NP.

Our computational results for $(\mathcal{D}, \mathbf{w})$-RECOMMENDATION are summarized in Table 1. We show that this problem is NP-hard, even when the weight function is linear (Theorem 3). On the algorithmic side, we provide a polynomial-time approximation scheme (PTAS) and a 5-approximation algorithm for the linear weight function (Theorems 4 and 5), and an approximation scheme for non-linear weights (Theorem 6).

Theorem 2 (Exact Algorithms). ( $\mathcal{D}, \mathbf{w})$-RECOMMENDATION is solvable in polynomial time when $\mathrm{w}$ is linear and $\mathcal{D}$ is

${ }^{3}$ Here, 'time taken' includes the time spent in thinking about which alternative to move as well as actually carrying out the move. 
either (a) k-mixture Plackett-Luce $(k$-PL) with $k=1$, (b) $k$ mixture Mallows model ( $k$-MM) with $k=1$, or $(c)$ a uniform distribution with support size $n \leq 2$.

Theorem 3 (Hardness results). ( $\mathcal{D}, \mathbf{w})$-RECOMMENDATION is NP-complete even when $\mathrm{w}$ is linear and $\mathcal{D}$ is either $(a) k$ mixture Plackett-Luce model ( $k$-PL) for $k=4$, (b) $k$-mixture Mallows model ( $k$-MM) for $k=4$, or (c) a uniform distribution over $n=4$ linear orders.

Theorem 4 (PTAS). ( $\mathcal{D}, \mathbf{w})$-RECOMMENDATION admits a polynomial time approximation scheme (PTAS) when $\mathrm{w}$ is linear and $\mathcal{D}$ is either (a) $k$-mixture Plackett-Luce model ( $k$-PL) for $k \in \mathbb{N}$, (b) $k$-mixture Mallows model $(k-\mathrm{MM})$ for $k \in \mathbb{N}$, or (c) a uniform distribution (Unif).

The PTAS in Theorem 4 is quite complicated and is primarily of theoretical interest (indeed, for any fixed $\varepsilon>0$, the running time of the algorithm is $m^{2^{\tilde{\mathcal{O}}(1 / \varepsilon)}}$, making it difficult to be applied in experiments). A simpler and more practical algorithm (although with a worse approximation) is based on the well-known Borda count voting rule (Theorem 5).

Theorem 5 (5-approximation). ( $\mathcal{D}, \mathbf{w})$-RECOMMENDATION admits a polynomial time 5-approximation algorithm when $\mathbf{w}$ is linear and $\mathcal{D}$ is either (a) k-mixture Plackett-Luce model ( $k$-PL) for $k \in \mathbb{N}$, (b) $k$-mixture Mallows model ( $k$-MM) for $k \in \mathbb{N}$, or (c) a uniform distribution (Unif).

Our next result (Theorem 6) provides an approximation guarantee for $(\mathcal{D}, \mathbf{w})$-RECOMMENDATION that applies to nonlinear weight functions, as long as they are "close" to the linear weight function in the following sense:

Definition 6 (Closeness-of-weights). A weight function $\mathbf{w}$ is said to be $(\alpha, \beta)$-close to another weight function $\mathbf{w}^{\prime}$ if there exist $\alpha, \beta \geq 1$ such that for every $\ell \in[m-1]$, we have

$$
\mathbf{w}^{\prime}(\ell) / \beta \leq \mathbf{w}(\ell) \leq \alpha \mathbf{w}^{\prime}(\ell) .
$$

For any (possibly non-linear) weight function $\mathbf{w}$ that is $(\alpha, \beta)$ close to the linear weight function $\mathbf{w}_{\text {lin }}$, Theorem 6 provides an $\alpha \beta$-approximation scheme for $(\mathcal{D}, \mathbf{w})$ RECOMMENDATION.

Theorem 6 (Approximation for general weights). Given any $\varepsilon>0$ and any weight function $\mathbf{w}$ that is $(\alpha, \beta)$-close to the linear weight function $\mathbf{w}_{\text {lin }}$, there exists an algorithm that runs in time $m^{2^{\tilde{\mathcal{O}}(1 / \varepsilon)}}$ and returns a linear order $\sigma$ such that

$$
\begin{aligned}
& \mathbb{E}_{\tau \sim \mathcal{D}}\left[\operatorname{time}_{\mathbf{w}}(\sigma, \tau)\right] \leq \alpha \beta(1+\varepsilon) \mathbb{E}_{\tau \sim \mathcal{D}}\left[\operatorname{time}_{\mathbf{w}}\left(\sigma^{*}, \tau\right)\right], \\
& \text { where } \sigma^{*} \in \arg \min _{\sigma^{\prime} \in \mathcal{L}(A)} \mathbb{E}_{\tau \sim \mathcal{D}}\left[\operatorname{time}_{\mathbf{w}}\left(\sigma^{\prime}, \tau\right)\right]
\end{aligned}
$$

Remark 2. Notice that the PTAS of Theorem 4 is applicable for any affine weight function $\mathbf{w}_{\text {aff }}=c \cdot \mathbf{w}_{\text {lin }}+d$ for some fixed constants $c, d \in \mathbb{N}$. As a result, the approximation guarantee of Theorem 6 also extends to any weight function that is $(\alpha, \beta)$ close to some affine weight function.

\section{Experimental Results}

We perform two sets of experiments on Amazon Mechanical Turk (MTurk). The first set of experiments (Section 5.1) is aimed at identifying the sorting strategies of the users as well as a model of their drag-and-drop behavior. The observations from these experiments directly motivate the formulation of our theoretical model, which we have already presented in Section 4. The second set of experiments (Section 5.2) is aimed at evaluating the practical usefulness of our approach.

In both sets of experiments, the crowdworkers were asked to sort in increasing order randomly generated lists of numbers between 0 and 100 . Sections 5.1 and 5.2 provide details about the length of the lists and how they are generated.

In each experiment, the task length was advertised as 10 minutes, and the payment offered was $\$ 0.25$ per task. The crowdworkers were provided a user interface (see Figure 1) that allows for drag-and-drop operations. To ensure data quality, we removed those workers from the data who failed to successfully order the integers more than $80 \%$ of the time, or did not complete all the polls. We also removed the workers with high variance in their sorting time; in particular, those with coefficient of variation above the $80^{\text {th }}$ percentile. The reported results are for the workers whose data was retained.

\subsection{Identifying User Behavior}

To identify user behavior, we performed two experiments: (a) RANK 10, where each crowdworker participated in 20 polls, each consisting of a list of 10 integers (between 0 and 100) generated uniformly at random, and (b) RANK5, which is a similar task with 30 polls and lists of length 5. In each poll, we recorded the time taken by a crowdworker to move an alternative (via drag-and-drop operation) and the number of positions by which the alternative was moved. After the initial pruning (as described above), we retained 9840 polls submitted by 492 workers in the RANK 10 experiment, and 10320 polls submitted by 344 workers retained in the RANK 5 experiment. Table 2 summarizes the aggregate statistics. Our observations are discussed below.

Sorting behavior. Our hypothesis regarding the ranking behavior of human crowdworkers was that they use (some combination of) natural sorting algorithms such as selection sort or insertion sort (Section 3.1). To test our hypothesis, we examined the fraction of the drag-and-drop operations that coincided with an iteration of selection/insertion sort. (Given a ranking $\sigma$, a drag-and-drop operation on $\sigma$ coincides with selection/insertion sort if the order of alternatives resulting from the drag-and-drop operation exactly matches the order of alternatives when one iteration of either selection or insertion sort is applied on $\sigma$.) We found that, on average, $\frac{2.21}{2.91}=76 \%$ of all drag-and-drop operations in RANK5 (and $\frac{5.09}{7.69}=66.2 \%$ in the RANK10) coincided with selection/insertion sort.

Drag-and-drop behavior. To identify the drag-and-drop behavior of the users, we plot the time-to-rank as a function of the total number of positions by which the alternatives are moved in each poll (Figure 3). Recall from Remark 1 that for an ideal user who uses only insertion/selection sort, the latter quantity is equal to $\mathrm{d}_{\mathrm{kt}}(\sigma, \tau)$. Our hypothesis was that the sorting time varies linearly with the total number of dragand-drop operations (\#moves) and the Kendall's Tau distance $\left(\mathrm{d}_{\mathrm{kt}}(\sigma, \tau)\right)$. To verify this, we used linear regression with timeto-rank (or sorting time) as the target variable and measured the mean squared error (MSE) using 5-fold cross-validation 
Proceedings of the Twenty-Eighth International Joint Conference on Artificial Intelligence (IJCAI-19)

\begin{tabular}{|c|c|c|c|c|c|c|}
\hline & \multicolumn{3}{|c|}{ RANK10 } & \multicolumn{3}{|c|}{ RANK5 } \\
\cline { 2 - 7 } & Mean & Median & Std. Dev. & Mean & Median & Std. Dev. \\
\hline Sorting time & 24.41 & 22.65 & 9.12 & 7.75 & 6.99 & 3.54 \\
\hline Total number of drag-and-drop operations & 7.69 & 8 & 1.8 & 2.91 & 3 & 1.13 \\
\hline Total number of positions moved during drag-and-drop operations & 22.59 & 23 & 5.59 & 5.05 & 5 & 2.01 \\
\hline Number of operations coinciding with selection/insertion sort & 5.09 & 6 & 2.28 & 2.21 & 2 & 1.06 \\
\hline Kendall's Tau distance between the initial and final rankings & 22.55 & 22 & 5.6 & 5.04 & 5 & 2.01 \\
\hline
\end{tabular}

Table 2: Summary of the user statistics recorded in the experiments in Section 5.1.

\begin{tabular}{|c|c|c|c|c|c|c|}
\hline \multirow{2}{*}{ Dataset } & \multirow{2}{*}{$\begin{array}{c}\text { Avg. MSE } \\
\text { (in seconds }\end{array}{ }^{2}$ ) } & \multirow{2}{*}{$\begin{array}{c}\text { Avg. MSE } \\
\text { (in seconds) }\end{array}$} & Avg. Sorting Time & \multicolumn{3}{|c|}{ Number of users based on their best-fit model } \\
\cline { 5 - 7 } & (in seconds) & Only $d_{\mathrm{kt}}$ & Only \#moves & Both $\mathrm{d}_{\mathrm{kt}}$ and \#moves \\
\hline RANK10 & 42.98 & 6.56 & 24.41 & 217 & 199 & 76 \\
\hline RANK5 & 7.74 & 2.78 & 7.75 & 138 & 180 & 26 \\
\hline
\end{tabular}

Table 3: Average 5-fold cross-validation MSE over all workers using the best model for each worker, and the number of users for which each of the models was identified to be the best. \# moves is the number of times alternatives are moved using selection or insertion sort.
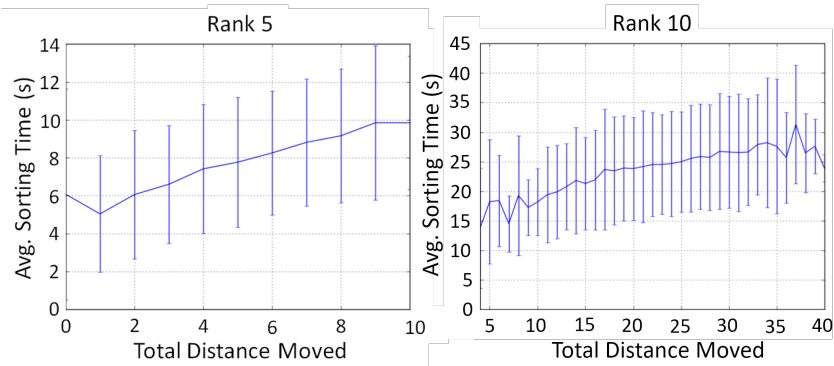

Figure 3: Relationship between the number of positions moved and the total sorting time for RANK5 (left) and RANK10 (right).

for three different choices of independent variables: (1) Only $\mathrm{d}_{\mathrm{kt}}$, (2) only \#moves, and (3) both $\mathrm{d}_{\mathrm{kt}}$ and \#moves. For each user, we picked the model with the smallest MSE (see Table 3 for the resulting distribution of the number of users). We found that the predicted drag-and-drop times (using the best-fit model for each user) are, on average, within $\frac{6.56}{24.41}=26.8 \%$ of the observed times for RANK10 and within $\frac{2.78}{7.75}=35.8 \%$ for RANK5.

\subsection{Evaluating the Proposed Framework}

To evaluate the usefulness of our framework, we compared a random recommendation strategy with one that forms an increasingly accurate estimate of users' preferences with time. Specifically, we first fix the ground truth ranking of 10 alternatives consisting of randomly generated integers between 0 and 100. Each crowdworker then participates in two sets of 10 polls each. In one set of polls, the crowdworkers are provided with initial rankings generated by adding independent Gaussian noise to the ground truth (to simulate a random recommendation strategy), and their sorting times are recorded.

In the second set of polls, the recommended set of alternatives is the same as under the random strategy but ordered order to a Borda ranking. Specifically, the ordering in the $k^{\text {th }}$ iteration is determined by the Borda ranking aggregated from the previous $k-1$ iterations.

Figure 4 shows the average sorting time of the crowdworkers as a function of the index of the polls under two different
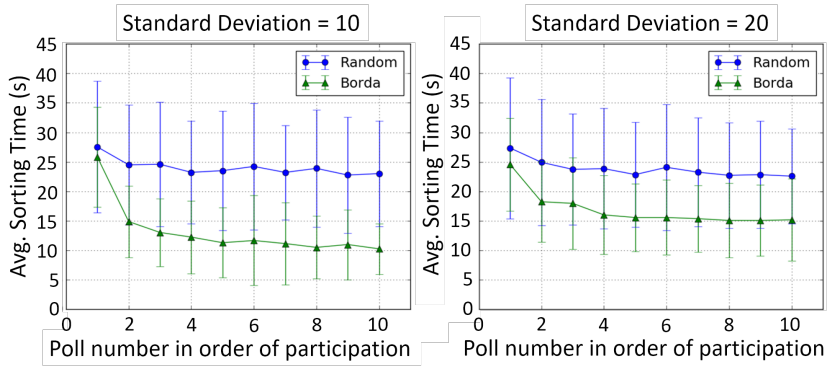

Figure 4: Relationship between sorting time and the number of polls completed by the users for $\mathrm{std} d \mathrm{dev}=10$ (left) and std dev=20 (right).

noise settings: std. dev. $=10$ and std. dev. $=20$.

We observe that Borda recommendation strategy (in green) provides a significant reduction in the sorting time of the users compared to the random strategy (in blue). Indeed, the sorting time of the users is reduced by up to $50 \%$, thus validating the practical usefulness of our framework. Note that the reduction in sorting time is not due to increasing familiarity with the interface. This is because the average sorting time for the random strategy remains almost constant throughout the duration of the poll.

\section{Future Work}

Our work opens up a number of directions for future research. First, it would be interesting to analyze the complexity of the recommendation problem for other distance measures, e.g., Ulam distance. Second, it would be interesting to analyze the effect of cognitive biases such as the framing effect [Tversky and Kahneman, 1981] and list position bias [Lerman and Hogg, 2014] on the recommendation problem. Progress in this direction can, in turn, have implications on the fairness of recommendation algorithms.

\section{Acknowledgments}

We are grateful to IJCAI-19 reviewers for their helpful comments. This work is supported by NSF \#1453542 and ONR \#N00014-17-1-2621. 


\section{References}

[Agarwal, 2016] Shivani Agarwal. On Ranking and Choice Models. In Proceedings of the Twenty-Fifth International Joint Conference on Artificial Intelligence, pages 40504053, 2016.

[Albert and Tullis, 2013] William Albert and Thomas Tullis. Measuring the User Experience: Collecting, Analyzing, and Presenting Usability Metrics. Newnes, 2013.

[Awasthi et al., 2014] Pranjal Awasthi, Avrim Blum, Or Sheffet, and Aravindan Vijayaraghavan. Learning Mixtures of Ranking Models. In Proceedings of Advances in Neural Information Processing Systems, pages 2609-2617, 2014.

[Bevan et al., 2015] Nigel Bevan, James Carter, and Susan Harker. ISO 9241-11 revised: What have we Learnt about Usability Since 1998? In International Conference on Human-Computer Interaction, pages 143-151, 2015.

[Blum et al., 2004] Avrim Blum, Jeffrey Jackson, Tuomas Sandholm, and Martin Zinkevich. Preference Elicitation and Query Learning. Journal of Machine Learning Research, 5:649-667, 2004.

[Boutilier, 2013] Craig Boutilier. Computational Decision Support: Regret-Based Models for Optimization and Preference Elicitation. In P. H. Crowley and T. R. Zentall, editors, Comparative Decision Making: Analysis and Support Across Disciplines and Applications. Oxford University Press, 2013.

[Braverman and Mossel, 2008] Mark Braverman and Elchanan Mossel. Noisy Sorting Without Resampling. In Proceedings of the Nineteenth Annual ACM-SIAM Symposium on Discrete Algorithms, pages 268-276, 2008.

[Busa-Fekete et al., 2014] Róbert Busa-Fekete, Eyke Hüllermeier, and Balázs Szörényi. Preference-Based Rank Elicitation using Statistical Models: The Case of Mallows. In Proceedings of the 31st International Conference on International Conference on Machine Learning, pages II:1071-1079, 2014.

[Conitzer and Sandholm, 2002] Vincent Conitzer and Tuomas Sandholm. Vote Elicitation: Complexity and StrategyProofness. In Eighteenth National Conference on Artificial Intelligence, pages 392-397, 2002.

[Guiver and Snelson, 2009] John Guiver and Edward Snelson. Bayesian Inference for Plackett-Luce Ranking Models. In Proceedings of the 26th Annual International Conference on Machine Learning, ICML-09, pages 377-384, 2009.

[Kendall, 1938] Maurice G Kendall. A New Measure of Rank Correlation. Biometrika, 30(1/2):81-93, 1938.

[Khetan and Oh, 2016] Ashish Khetan and Sewoong Oh. Data-Driven Rank Breaking for Efficient Rank Aggregation. Journal of Machine Learning Research, 17(193):1-54, 2016.

[Laskowski et al., 2004] Sharon J Laskowski, Marguerite Autry, John Cugini, and William Killam. Improving the Usability and Accessibility of Voting Systems and Products. NIST Special Publication, 500:256, 2004.
[Lerman and Hogg, 2014] Kristina Lerman and Tad Hogg. Leveraging Position Bias to Improve Peer Recommendation. PloS one, 9(6):e98914, 2014.

[Li et al., 2019] Haoming Li, Sujoy Sikdar, Rohit Vaish, Junming Wang, Lirong Xia, and Chaonan Ye. Minimizing Time-to-Rank: A Learning and Recommendation Approach. arXiv preprint arXiv:1905.11984, 2019.

[Liu, 2011] Tie-Yan Liu. Learning to Rank for Information Retrieval. Springer, 2011.

[Lu and Boutilier, 2014] Tyler Lu and Craig Boutilier. Effective Sampling and Learning for Mallows Models with Pairwise-Preference Data. Journal of Machine Learning Research, 15:3963-4009, 2014.

[Luce, 1959] Robert Duncan Luce. Individual Choice Behavior: A Theoretical Analysis. Wiley, 1959.

[Mallows, 1957] Colin L. Mallows. Non-Null Ranking Model. Biometrika, 44(1/2):114-130, 1957.

[Negahban et al., 2017] Sahand Negahban, Sewoong Oh, and Devavrat Shah. Rank Centrality: Ranking from Pairwise Comparisons. Operations Research, 65(1):266-287, 2017.

[Plackett, 1975] Robin L. Plackett. The Analysis of Permutations. Journal of the Royal Statistical Society. Series C (Applied Statistics), 24(2):193-202, 1975.

[Procaccia and Shah, 2016] Ariel D Procaccia and Nisarg Shah. Optimal Aggregation of Uncertain Preferences. In Thirtieth AAAI Conference on Artificial Intelligence, pages 608-614, 2016.

[Soufiani et al., 2013] Hossein Azari Soufiani, David C Parkes, and Lirong Xia. Preference Elicitation For General Random Utility Models. In Proceedings of the TwentyNinth Conference on Uncertainty in Artificial Intelligence, pages 596-605, 2013.

[Tversky and Kahneman, 1981] Amos Tversky and Daniel Kahneman. The Framing of Decisions and the Psychology of Choice. Science, 211(4481):453-458, 1981.

[Xia, 2019] Lirong Xia. Learning and Decision-Making from Rank Data. Synthesis Lectures on Artificial Intelligence and Machine Learning. Morgan \& Claypool Publishers, 2019.

[Zhao and Xia, 2018] Zhibing Zhao and Lirong Xia. Composite Marginal Likelihood Methods for Random Utility Models. In Proceedings of the 35th International Conference on Machine Learning, pages 5922-5931, 2018.

[Zhao et al., 2016] Zhibing Zhao, Peter Piech, and Lirong Xia. Learning Mixtures of Plackett-Luce Models. In Proceedings of the 33rd International Conference on Machine Learning (ICML-16), 2016.

[Zhao et al., 2018] Zhibing Zhao, Haoming Li, Junming Wang, Jeffrey Kephart, Nicholas Mattei, Hui Su, and Lirong Xia. A Cost-Effective Framework for Preference Elicitation and Aggregation. In Proceedings of the 2018 Conference on Uncertainty in Artificial Intelligence (UAI), 2018. 